\title{
Demon Genes May Deform Common Syndromes: Collagen VI Gene Change in Down Syndrome Unifies the Medical and Molecular Approach to Hypermobility Disorders
}

\author{
Golder N. Wilson ${ }^{1 *}$ (D), Vijay S. Tonk ${ }^{2}$ \\ ${ }^{1}$ Department of Pediatrics, Texas Tech University Health Science Center, Lubbock and KinderGenome Medical Genetics, Dallas, \\ TX, USA \\ ${ }^{2}$ Departments of Pediatrics, Obstetrics \& Gynecology, and Pathology, Texas Tech University Health Science Center, Lubbock, TX, \\ USA \\ Email: ^golder.wilson@ttuhsc.edu
}

How to cite this paper: Wilson, G.N. and Tonk, V.S. (2022) Demon Genes May Deform Common Syndromes: Collagen VI Gene Change in Down Syndrome Unifies the Medical and Molecular Approach to Hypermobility Disorders. Journal of Biosciences and Medicines, 10, 1-7. https://doi.org/10.4236/jbm.2022.103001

Received: January 24, 2022

Accepted: February 28, 2022

Published: March 2, 2022

Copyright $\odot 2022$ by author(s) and Scientific Research Publishing Inc. This work is licensed under the Creative Commons Attribution International License (CC BY 4.0).

http://creativecommons.org/licenses/by/4.0/

(c) (i) Open Access

\begin{abstract}
Purpose: To alert the medical community that whole exome sequencing can find accessory gene changes in well-known syndromes that alter preventive health care and management. Meaning: A collagen type VI gene change adds muscle weakness, hypermobility, and dysautonomia concerns to usual management considerations for Down syndrome. Methods: Commercial whole exome sequencing combined with clinical interpretation of DNA sequence change added new considerations to patient management and parental counsel. Results: An 11-year-old child with the trisomy 21 form of Down syndrome who was evaluated for extraordinary joint laxity had a heterozygous collagen type VI aspartic to glutamic acid (COL6A3 c.6360 C >G p.Asp2120Glu) gene change found by whole exome sequencing. The DNA variant was qualified as having strong relevance to the enhanced hypermobility due to prior association of collagen 6 gene changes with myopathy. Conclusions: Dual diagnosis of Ehlers-Danlos syndrome was not assigned because the patient lacked criteria like bruising, unusual scars, or selected dysautonomia symptoms. The concept of a hypermobility spectrum offers advantages for management of its constituent conditions if clinically guided ascertainment and DNA diagnostics are employed.
\end{abstract}

\section{Keywords}

Hypermobility Spectrum Disorders, Down Syndrome, Ehlers-Danlos Syndrome, DNA Variant Qualification, Collagen Type VI DNA Change 


\section{Introduction}

The approach from general to particular, so essential for Linnaean taxonomy and medical diagnosis, has now been applied to hypermobility as the avatar of diseases like Ehlers-Danlos syndrome (EDS, [1] [2]). Unusual flexibility or hypermobility occurs in two major disease categories, one involving low muscle tone with less constraint of joints (intellectual disability-hypotonia disorders-[3]), the other involving laxity of the joint tissue itself (connective tissue dysplasias-[4] [5] [6]). Clinical recognition of hypermobility offers many benefits for patients, ranging from anticipatory guidance of joint injury and autonomic imbalance in its more benign forms [6] [7] to prevention of lethal cardiovascular complications in its extremes [8].

Another key to the recognition and differential diagnosis of hypermobility is appreciation of the many genes that must contribute to the synthesis and modulation of connective tissue [9], the medium that first enabled protists to become multicellular, the conveyer of messages that integrate cellular functions. Genetic control of this substrate for primal evolutionary transitions and present hypermobility disorders can now be defined by NextGen sequencing, alterations in code and molecular architecture detailing connective tissue faults in hypotonia, dysplasia, and aging. DNA analysis can refine the diagnosis and management of hypermobility as shown by the following patient presentation and discussion.

\section{Clinical Report}

An 11-year and 9-month-old girl was brought to genetics clinic by her mother for evaluation of Ehlers-Danlos syndrome after several physicians had commented on joint laxity and bowel issues beyond those expected for Down syndrome. She was adopted from Asia at age 2 with documentation of trisomy 21 but few records of gestational, medical, or family history besides premature delivery around 35 weeks and significant neonatal feeding difficulties. Her parents noticed remarkable flexibility with unusual postures from the beginning, persisting gastrointestinal reflux and constipation leading to a diagnosis of rectosigmoid aganglionosis. Prolonged recovery after surgery was complicated by enterocolitis, bloating, and constipation that was diagnosed as irritable bowel syndrome. Her mother reported slow healing around her ostomy site with slow weight gain aided by frequent small feedings and a high-protein diet. She had been diagnosed with autism in India and was followed for that diagnosis in developmental pediatrics, described as a happy child with limited language and a developmental level about half of normal at age 9 . Over the past year she became more irritable and withdrawn, resisting activities and using words like "hurt" or "ouch" when walking. She became more fatigued, sometimes sleeping 18 hours a day. Behavioral tantrums and outburst have worsened and she has been on risperidone with consideration of adding Intuniv by her pediatrician. Evaluation by rheumatology recognized hypermobility without autoimmune markers and wrist $\mathrm{X}$-rays documented a 2-year delay in bone age with lower bone density. 
Physical examination was complicated by resistant behaviors but showed a height of $115 \mathrm{~cm}$ ( $25^{\text {th }}$ centile), weight of $25 \mathrm{~kg}\left(50^{\text {th }}\right.$ centile), and head circumference of $46 \mathrm{~cm}$ ( $3^{\text {rd }}$ centile), all on the Down syndrome chart. She had the typical facial appearance with brachycephaly, epicanthal folds, overturned helices of small ears, down-turned corners of the mouth, and broad neck. Most striking was her hypermobility, still sitting in the $\mathrm{W}$ position and able to place her face prone on the table. Passive performance of 8 of the 9 Beighton maneuvers was easy and without pain, observation suggesting that she could place palms to floor without difficulty to give 9 of 9 . Her skin was soft but not velvety or lucent and was elastic based on the ability to raise thin, epidermal folds on her jaw and forearm of over 2 inches. She had no atrophic, papyraceous, or keloid scarring but did have some rough and raised skin around the ostomy site that may have been the keratosis described in patients with COL6-related Bethlem myopathy [10]. Lordosis but not scoliosis was evident on standing and her feet were flat with valgus positioning when walking. Muscle strength was not obviously decreased against resistance but hypotonia in addition to hypermobility was suggested by her persisting head lag when brought from supine and her slumping posture with slight scapular winging when sitting. She was able to play without obvious focal deficits, exhibiting some escape and hitting behaviors despite limited activity.

Added to her trisomy 21 chromosome analysis was whole exome sequencing conducted through the GeneDx ${ }^{\odot}$ Company using standard methods. Preliminary ascertainment of favorable out-of-pocket costs, consent for testing with revelation of incidental findings, and collection of blood or cheek swab samples were all coordinated with the help of GeneDx genetic counselors.

The collagen type VI gene change below was relayed to the family and interpreted as contributing to her hypermobility through muscle change [10] but not to a dual diagnosis of EDS. However, her implied joint pain, chronic fatigue, and irritable bowel syndrome were explained as well-documented consequences of reciprocal tissue laxity and dysautonomia in hypermobility disorders like EDS [6]. She lacked current symptoms of the postural orthostatic tachycardia or mast-cell activation disorder that can also be part of the dysautonomia, but they were described to the family as future possibilities. The mutation was proximal to the site for 5'-cleavage of COL6A3 that yields an endotrophin peptide, presumably not altering its dosage that has been related to certain cancers and metabolic syndrome [11]. Immediate management recommendations included annual clinic visits for broad medical monitoring, physical therapist-dietician consultation with institution of a low-impact core strength program, and probiotics with reduced dietary gluten and dairy products for her irritable bowel syndrome.

\section{Results}

A heterozygous DNA variant with nucleotide change c.6360C $>\mathrm{G}$ encoding a p.Asp2120Glu amino acid substitution in the collagen type VI alpha-3 chain (COL6A3) gene was the only alteration reported, microarray analysis not per- 
formed concurrently at the time of whole exome sequencing (2015). The mutation was qualified as a variant of uncertain significance by commercial report

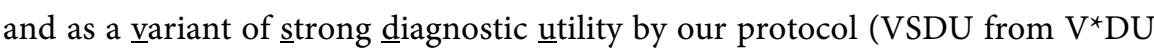
where $^{*}=\mathrm{N}$ for No, $\mathrm{U}$ for uncertain, $\mathrm{M}$ for moderate, $\mathrm{S}$ for strong, $\mathrm{E}$ for evidenced-[12]). The latter qualification reflected a $2+$ impact on protein structure because of the dramatic conformational differences between aspartic and glutamic acid [13] and its position in a conserved COL6A3 protein domain as annotated by GeneDx. The variant was and remains unlisted by ClinVar or normal population databases.

Additional pluses in our protocol could not be assigned for variant-symptom concordance in family members-parental samples were not obtained-but a plus was added because of plausible pathogenic mechanism: Known impact on muscle as shown by other COL6A3 gene mutations [10] suggests that this variant could augment tissue laxity by weakening surrounding muscular constraint. No additional DNA variants with synergistic pathogenic action $\left(\mathrm{V}^{*} \mathrm{DU} \underline{\mathrm{S}}\right)$ were reported that would augment diagnostic utility (the known flexibility of trisomy 21 might add a plus for the combined DNA result). A final MDna3+ medical diagnostic utility score was assigned using a clinically familiar and medically determined MDna1-4+ scale [12]. The lack of joint instability, bruising, unusual scars, or symptoms of autonomic imbalance besides bowel suppression related 3+ diagnostic utility to hypermobility rather than EDS. No secondary findings with other pathogenic action ( $\left.\mathrm{V}^{\star} \mathrm{DU} \underline{\mathrm{O}}\right)$ implied dual diagnoses.

\section{Discussion}

Among the $20 \%$ of women, $10 \%$ of men, and $40 \%$ of children who have hypermobility are an ill-defined proportion with life limitation from skeletal instability, injury, deformation, and pain [1] [2]. In many cases these complications of "double-jointedness" are accompanied by findings that differentiate types of hypermobility disorders, including conditions with hypotonia accompanying intellectual disability (as in chromosome disorders, [3]) and skin-skeletal-organ changes accompanying tissue laxity (as in connective tissue dysplasias, [4] [5] [6]). As with most common traits, hypermobility and its component disorders combine polygenic proposal with environmental disposition. Medical genomics is available to define composite genotypes, but holistic ascertainment and correlation are needed to understand their mediation as clinical outcomes.

Genomic characterization of hypermobility disorders requires the more lenient candidate gene approach used in prior gene mapping and present whole genome association studies [12]. Biochemical implications of DNA variance can guide preliminary qualification using amino acid conformational change predicted by Ramachandran plots [13]; structural considerations can promote candidacy when complex clinical pathways like those connecting tissue laxity with dysautonomia are not easily modelled by in vitro functional analyses. The latter should follow rather than precede consideration of DNA variant significance according to biochemical impact, electing gene change to official disease contri- 
bution by matching presumed clinical with defined molecular mechanisms.

This COL6-hypermobility-trisomy 21 conjunction spurs many thoughts including relationships of myopathy and hypermobility [10], roles of COL6 extracellular matrix biosynthesis/breakdown and inflammation (through transforming growth factor-beta pathways) in connective tissue dysplasias [11], molecular insights as to how COL6A3 (at 2q37.3) gene change would affect its usually stoichiometric assembly with COL6A1 and COL6A2 proteins (genes at 21q22.3) when the latter have extra copies due to trisomy 21.

Phenotypic characterization of hypermobility disorders is helped by the evolution of diagnostic criteria $[1,5]$ and by the development of instruments such as the forms that assess 80 history and 40 physical findings in EDS [6]. Combining the gestalt diagnosis of Down syndrome with the appreciation of typical minor anomalies and checklists for complications led to dramatic improvements in quality and length of life. Similar advantages for hypermobility disorders would come from coupling the prototypic skeletal and skin findings with reciprocal ones of vessel distensibility-lower body blood pooling (menorrhagia, pelvic congestion, varicosities, foot discoloration on standing) and its resulting adrenergic imbalance (tachycardia, allergy, anxiety, fatigue with suppression of bowel motility); the latter under-appreciated findings are present in 30 to 90 percent of EDS patients [6] [9]. Earlier recognition of EDS and other hypermobility spectrum disorders can validate pain-stress-anxiety as medical problems and initiate joint protection (braces, muscle-building exercise) and nutritional (salt, hydration, lower gluten-dairy intake) therapies.

Better delineation of disorders highlighted by hypermobility depends on combined descriptive and DNA diagnostic approaches, both optimized by clinical experience that in this case would not assign dual diagnoses of Down and EDS to our patient as done in a prior instance [14]. Consider hypermobility as trait first (even mild hypermobility can have dysautonomia symptoms-[7]), then as consequence in the many disorders with central hypotonia (augmented by myopathic gene change in this case), then as signal for associated findings in the connective tissue dysplasias (EDS considered but rejected in this case when criteria of scarring, bruising, valve prolapse, and certain autonomic symptoms were lacking).

A broad approach to hypermobility alerts to a host of tissue laxity and dysautonomia complications when recognized in childhood as a genetic predisposition and later as a consequence of aging. The chronic pain and fatigue that are too often attributed to malingering, the pain-related depression [15] and adrenaline-related anxiety that are misread as mental illness, can then be diagnosed as medical issues and treated appropriately. It is not unrealistic to think that the gene networks altered in hypermobility, when appropriately correlated, could eventually be reoriented by gene/stem cell correction in the way that exercise can presently reorient lax tissue by strengthening its surrounding muscle.

\section{The Authors Certify}

1) I and Dr. Vijay Tonk, my co-author, have read and agreed to the article 
content and are accountable for all parts of the manuscript;

2) The article is not under consideration for publication elsewhere.

\section{Conflicts of Interest}

The authors declare no conflicts of interest regarding the publication of this paper.

\section{References}

[1] Castori, M., Tinkle, B., Levy, H., Grahame, R., Malfait, F. and Hakim, A. (2017) A Framework for the Classification of Joint Hypermobility and Related Conditions. American Journal of Medical Genetics Part C: Seminars in Medical Genetics, 175, 148-157. https://doi.org/10.1002/ajmg.c.31539

[2] Tinkle, B.T. and Levy, H.P. (2019) Symptomatic Joint Hypermobility: The Hypermobile Type of Ehlers-Danlos syndrome and the Hypermobility Spectrum Disorders. Medical Clinics of North America, 103, 1021-1033. https://doi.org/10.1016/j.mcna.2019.08.002

[3] Wyandt, H.E., Wilson, G.N. and Tonk, V.S. (2017) Gene and Genome Sequencing: Interpreting Genetic Variation at the Nucleotide Level. In: Human Chromosome Variation: Heteromorphism, Polymorphism, and Pathogenesis, 2nd Edition, Springer, Singapore, 419-454. https://doi.org/10.1007/978-981-10-3035-2 11

[4] McKusick, V.A. (1955) Heritable Disorders of Connective Tissue. I. The Clinical Behavior of Hereditary Syndromes. Journal of Chronic Disease, 2, 491-499. https://doi.org/10.1016/0021-9681(55)90148-X

[5] Tinkle, B., Castori, M., Berglund, B., et al. (2017) Hypermobile Ehlers-Danlos Syndrome (a.k.a. Ehlers-Danlos Syndrome Type III and Ehlers-Danlos Syndrome Hypermobility Type): Clinical Description and Natural History. American Journal of Medical Genetics Part C: Seminars in Medical Genetics, 175, 48-69. https://doi.org/10.1002/ajmg.c.31538

[6] Wilson, G. (2019) Clinical Analysis Supports Articulo-Autonomic Dysplasia as a Unifying Pathogenic Mechanism in Ehlers-Danlos Syndrome and Related Conditions. The Journal of Bioscience and Medicine, 7, 149-168. https://doi.org/10.4236/jbm.2019.76010

[7] Gazit, Y., Nahir, A.M., Grahame, R. and Jacob, G. (2003) Dysautonomia in the Joint Hypermobility Syndrome. The American Journal of Medicine, 115, 33-40. https://doi.org/10.1016/S0002-9343(03)00235-3

[8] Byers, P.H., Belmont, J., Black, J., et al. (2017) Diagnosis, Natural History, and Management in Vascular Ehlers-Danlos Syndrome. American Journal of Medical Genetics Part C: Seminars in Medical Genetics, 175, 40-47. https://doi.org/10.1002/ajmg.c.31553

[9] Wilson, G.N. (2019) Genomic Analysis of 727 Patients with Ehlers-Danlos Syndrome I: Clinical Perspective Relates 23 Genes to a Maternally Influenced Arthritis-Adrenaline Disorder. Journal of Biosciences and Medicines, 7, 181-204. https://doi.org/10.4236/jbm.2019.712015

[10] Foley, A.R., Mohassel, P., Donkervoort, S., et al. (2021) Collagen VI-Related Dystrophies. In: Adam, M.P., Ardinger, H.H., Pagon, R.A., et al., Eds., GeneReviews ${ }^{\infty}$ University of Washington, Seattle, 1993-2021.

[11] Scherer, P.E. and Gupta, O.T. (2021) Endotrophin: Nominated for Best Supporting Actor in the Fibro-inflammatory Saga. eBioMedicine, 69, Article ID: 103447. 
https://doi.org/10.1016/j.ebiom.2021.103447

[12] Wilson, G.N. and Tonk, V.S. (2021) A Protocol for Qualifying DNA Variants Associated with Complex Diseases like Ehlers-Danlos Syndrome. GenProtocols. https://genprotocols.genengnews.com/protocols/a-protocol-for-qualifying-dna-vari ants-associated-with-complex-diseases-like-ehlers-danlos-syndrome-/15

[13] Tam, B., Sinha, S. and Wang, S.M. (2020) Combining Ramachandran Plot and Molecular Dynamics Simulation for Structural-Based Variant Classification: Using TP53 Variants as Model. Computational and Structural Biotechnology Journal, 18, 4033-4039. https://doi.org/10.1016/j.csbj.2020.11.041

[14] Buterbaugh, A., Mroczkowski, H.J., Shankar, S.P. and Visootsak, J. (2013) Contribution of Family History in Co-Occurring Down Syndrome and Ehlers-Danlos Syndrome. Annals of Paediatric Rheumatology, 2, 165-167.

https://doi.org/10.5455/apr.081820131456

[15] Pizzo, P.A. (2013) Lessons in Pain Relief-A Personal Postgraduate Experience. The New England Journal of Medicine, 369, 1092-1093.

https://doi.org/10.1056/NEJMp1306467 